\title{
Eficacia de la vancomicina en polvo en la profilaxis de infección postquirúrgica en cirugía de columna
}

\author{
Effectiveness of vancomycin powder in the prophylaxis of infection post-surgical in spine surgery
}

\begin{abstract}
Gustavo Caldera Hernández, * Roberto IIdefonso Recillas Huante,‡ Normando Emmanuel Cob Garma§
*Médico adscrito a la División de Traumatología y Ortopedia, Módulo de Cirugía de Columna en Centro Médico Nacional de Occidente del Instituto Mexicano del Seguro Social; Guadalajara, Jalisco. Especialidad en Traumatología y Ortopedia, Cirugía de Columna; FFellowship en Cirugía de Columna en Centro Médico Nacional de Occidente del Instituto Mexicano del Seguro Social; Guadalajara, Jalisco. Especialidad en Traumatología y Ortopedia, Fellowship en

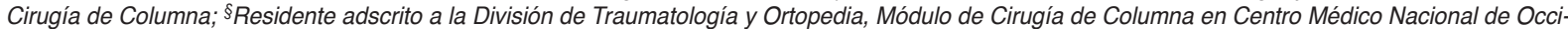
dente del Instituto Mexicano del Seguro Social; Guadalajara, Jalisco. Especialidad en Traumatología y Ortopedia.
\end{abstract}

\section{Resumen}

Por las capacidades farmacodinámicas y sus propiedades a nivel de la membrana celular, la vancomicina es considerada uno de los glucopéptidos de uso clínico como tratamiento de primera línea frente a la mayoría de los gérmenes Gram positivo. En cuanto a la vía tópica, existe evidencia de que la aplicación intraherida de antibióticos puede minimizar la infección local después de la cirugía. En los procedimientos realizados a nivel de la columna, la vancomicina ha cobrado importancia en su uso tópico como profilaxis. Objetivo: Analizar la eficacia de la vancomicina tópica en la prevención de infecciones a nivel de herida quirúrgica en la cirugía vertebral. Material y métodos: Se realizó un estudio de cohortes no aleatorizado, observacional y comparativo de 120 pacientes con cirugías instrumentadas de columna cervical, torácica y lumbar en el periodo comprendido entre enero 2019 a enero del 2020. En 60 casos con colocación de $1 \mathrm{~g}$ de vancomicina tópica, y 60 no, en forma no aleatorizada. Se realizó un seguimiento de los pacientes durante los primeros 90 días postquirúrgicos. Resultados: La relación entre vancomicina-infección no fue estadísticamente significativa, se identificaron cinco casos de infección de herida en el grupo sin vancomicina, se encontró que el factor edad menor de 50 años y patología traumática presenta un RR de 0.5833 y 0.2262 respectivamente aunque $\sin p \leq 0.05$, otro hallazgo fue el RR 2.0208 en un nivel lumbar instrumentado pero sin diferencia significativa; las principales características en los cinco pacientes con infección en la herida solo estuvieron presentes en el grupo sin vancomicina, identificando su edad, tipo de patología, nivel instrumentado y comorbilidades presentes. Conclusiones: No existe significancia estadística en nuestro estudio de que la vancomicina tópica juegue un papel fundamental en la prevención de infecciones a nivel de herida quirúrgica en la cirugía vertebral. Sin embargo, es importante notar que todos los pacientes infectados en este trabajo pertenecieron al grupo B (sin uso de vancomicina).

Palabras clave: Infección de sitio quirúrgico, instrumentación vía anterior, instrumentación vía posterior, vancomicina en polvo

\begin{abstract}
Due to its pharmacodynamic capacities and its properties at the level of the cell membrane, Vancomycin is considered one of the glycopeptides in clinical use as a first-line treatment against most Gram positive germs. Currently regarding the topical route, there is evidence that intra-wound application of antibiotics can minimize local infection after surgery. In procedures performed at the spinal level, vancomycin has gained importance in its topical use as prophylaxis. Objective: To analyze the efficacy of topical vancomycin in the prevention of infections at the surgical wound in vertebral surgery. Material and methods: A non-randomized, observational and comparative cohort study was carried out in 120 patients with instrumented cervical, thoracic and lumbar spine surgeries in the period from January 2019 to January 2020. In 60 cases with placement of $1 \mathrm{gr}$ of topical vancomycin, and 60 not, in a non-randomized way. The patients were followed up during the first 90 days after surgery. Results: The relationship between vancomycin infection was not statistically significant, identified five cases of wound infection in the group without vancomycin, it was found that the factor age less than 50 years and traumatic pathology presents an $R R$ of (0.5833) and (0.2262) respectively, although without $p \leq 0.05$, another finding was the $R R(2.0208)$ in a lumbar level instrumented but without significant difference; the main characteristics in the 5 patients with wound infection were only present in the group without vancomycin, identifying their age, type of pathology, instrumented level and present comorbidities. Conclusions: There is no statistical significance in our study that topical vancomycin play a fundamental role in the prevention of infection at the surgical wound in spinal surgery. However, it is important to note that all infected patients in this work belonged to group $B$ (without use of vancomycin).
\end{abstract}

Keywords: Surgical site infection, anterior instrumentation, posterior instrumentation, vancomycin powder.

Correspondencia:

Dr. Normando Emmanuel Cob Garma

E-mail: normandocobgarma@gmail.com

Citar como: Caldera HG, Recillas HRI, Cob GNE. Eficacia de la vancomicina en polvo en la profilaxis de infección postquirúrgica en cirugía de co-

Recibido: 04-02-2021. Aceptado: 08-02-2021. lumna. Orthotips. 2021; 17 (1): 25-31. https://dx.doi.org/10.35366/99164 


\section{Introducción}

El advenimiento de procesos infecciosos en cirugía de columna se considera en la actualidad un importante problema de salud. Su incidencia varía en función de múltiples factores, y se estima que va del 2 al $13 \%$ de los pacientes sometidos a cirugías de columna. ${ }^{1-3}$

La aplicación de vancomicina dentro del sitio quirúrgico se consolida como una alternativa novedosa para la prevención de las infecciones del sitio quirúrgico. Debido a un bajo costo, amplia disponibilidad y fácil aplicación, va ganando creciente aceptación entre los cirujanos de columna, basándose en las recomendaciones establecidas por la Sociedad de Columna de Norte América (NASS). ${ }^{4-7}$

Los microorganismos más frecuentes en infecciones a nivel de herida quirúrgica son $S$. epidermidis y $S$. aureus, los cuales son sensibles al espectro de la vancomicina tópica, por lo que estudios actuales sustentan la asociación en la reducción de la tasa de infecciones empleando el antibiótico vía intralesional. ${ }^{8-10}$

El objetivo del presente estudio es determinar la eficacia de la vancomicina tópica en la prevención de infecciones a nivel de herida quirúrgica en la cirugía vertebral. ${ }^{11-20}$

En Latinoamérica existe evidencia científica, con base en estudios controlados, de que la aplicación de vancomicina en polvo dentro de la herida se asocia con una reducción significativa del riesgo de infecciones del sitio quirúrgico, sin incrementar el de pseudoartrosis o de efectos adversos. Sin embargo, se requieren estudios controlados y aleatorizados, con el fin de confirmar los presentes resultados y realizar recomendaciones más certeras. ${ }^{19}$

En México se reporta que la prevalencia para infección de herida quirúrgica es de $1.5 \%$ en pacientes que recibieron vancomicina y de $5.3 \%$ en el grupo control. Los factores asociados a la presencia de infecciones fueron la edad, las instrumentaciones de más de seis niveles y la prolongación del tiempo quirúrgico. ${ }^{20}$

\section{Material y métodos}

Se realizó un estudio de cohortes no aleatorizado, observacional y comparativo. Se analizaron prospectivamente a 120 pacientes con cirugías instrumentadas de columna cervical, torácica o lumbar en el periodo comprendido entre enero 2019 a enero del 2020. En 60 casos se colocó $1 \mathrm{~g}$ de vancomicina tópica, y en 60 no, en forma no aleatorizada, según la preferencia de cada cirujano. Se realizó un seguimiento de los pacientes durante los primeros 90 días postquirúrgicos.

Se identificaron como pacientes del grupo A aquéllos que recibieron un ámpula de $1 \mathrm{~g}$ de vancomicina en polvo sobre el lecho de la herida quirúrgica por debajo del plano muscular previo al cierre del músculo y fascia (Figuras 1 y 2). En el grupo B no se aplicó vancomicina local. Todos los pacientes recibieron simultáneamente $1 \mathrm{~g}$ de cefalotina endovenosa 30 minutos antes de la incisión en piel, y continuaron con este antibiótico cada ocho horas durante las primeras 24 horas postquirúrgicas por protocolos hospitalarios de manejo antimicrobiano. ${ }^{11}$

Se incluyeron los pacientes operados con instrumentaciones de columna cervical, toracolumbar, por patologías degenerativas y traumáticas, con o sin comórbidos agregados.

Se excluyeron los pacientes con patología tumoral, infecciosa, alérgicos a la vancomicina y los pacientes con antecedentes de cirugías espinales previas.

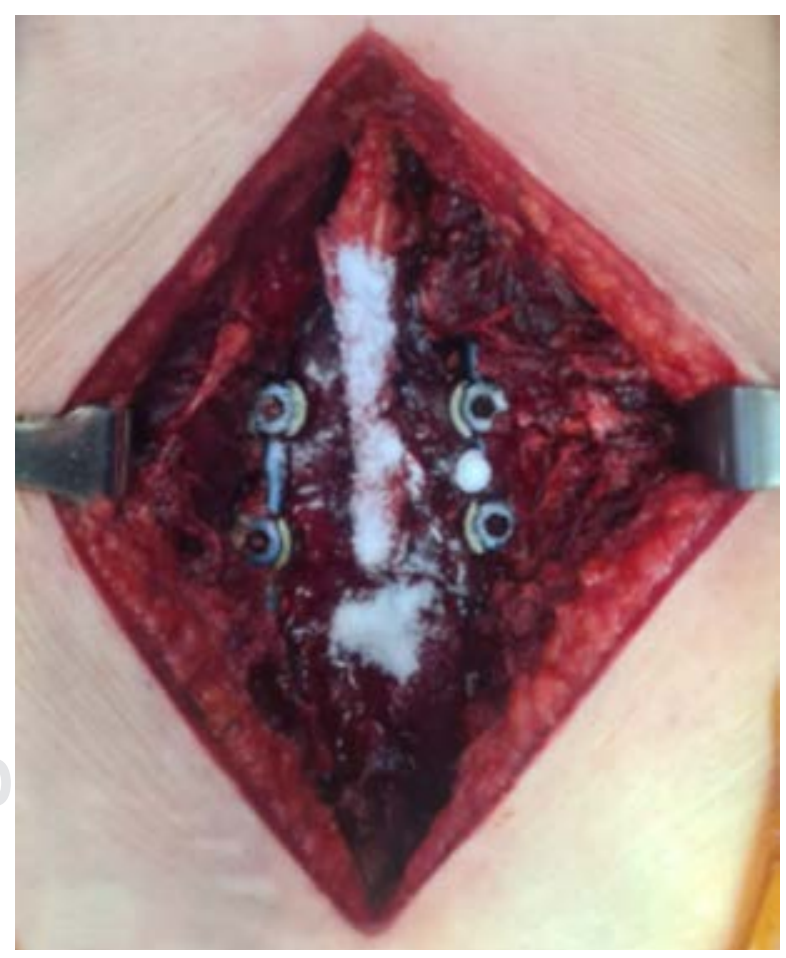

Figura 1: Paciente masculino, sometido a cirugía lumbar por fractura estallido L2, se aprecia colocación de polvo vancomicina $500 \mathrm{mg}$ una vez realizada la fijación transpedicular en fondo de herida. 


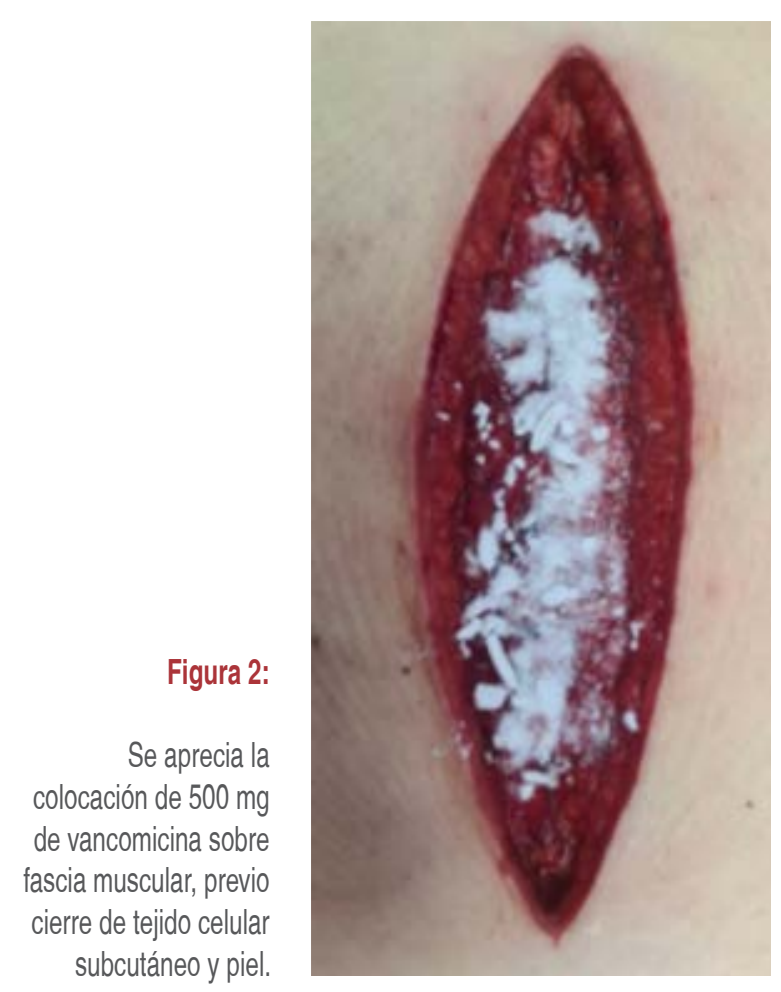

Hubo diferencias demográficas, rangos de edad y de comorbilidades, no así de la técnica quirúrgica entre el grupo que recibió y el que no recibió vancomicina.

El seguimiento de los pacientes fue presencial, controlando clínicamente la herida operatoria y signos relacionados con infección como fiebre, eritema o inflamación de la herida quirúrgica y dolor de características atípicas.

Definimos como «infecciones tempranas» al grupo que incluye infecciones agudas y subagudas, por lo cual incluyeron todas las infecciones que se manifestaron dentro de los tres meses posteriores a la intervención. De igual forma, las infecciones superficiales fueron definidas como aquéllas en las que los datos clínicos de infección se localizaron por arriba de la fascia y tejido celular subcutáneo, y profundas como aquéllas en las que se demostraron datos de infección por debajo de estos tejidos.

Los datos fueron volcados a una planilla de Excel (Microsoft 2013) y analizados con el programa de análisis estadístico Epi Info CDC. La estadística inferencial se realizó utilizando Fisher y $R R$ (riesgo relativo) para la comparación entre ambos grupos de las variables cualitativas. Se aceptaron como datos estadísticamente significativos valores de $p \leq 0.05$.

\section{Resultados}

Un total de 120 pacientes fueron incluidos y estudiados, 60 pacientes pertenecientes al grupo $A$ (uso de vancomicina) y el grupo $B, 60$ sin uso de vancomicina. Dentro de los pacientes del grupo A (Tabla 1), se encontró una edad promedio 55.1666 con DE de 14.9056, y en el grupo B 60.1666 con DE de 14.0619. En el aspecto del sexo, se encontró predominancia por el sexo masculino en el grupo con vancomicina, en el grupo sin vancomicina el sexo femenino fue el de mayor predominancia.

En ambos grupos, la región lumbar fue el segmento con mayor número de casos quirúrgicos (grupo $\mathrm{A}$ con un total de 48 casos vs 49 en el grupo B). En relación con la patología, el grupo A reportó predominio en patologías traumáticas en comparación al grupo $\mathrm{B}$, donde hubo una predominancia por la degenerativa (35 casos contra 32, respectivamente). El comórbido mayormente reportado en el grupo de vancomicina fue diabetes mellitus con un total de 27 personas, caso contrario en el grupo sin vancomicina un total de 24 .

En nuestro estudio, se reportaron cinco casos de infección de herida quirúrgica, estos cinco pertenecían al grupo B sin vancomicina (Tabla 2). De estos casos, tres fueron hombres y dos mujeres. Sólo hubo un caso de infección tipo profunda-tardía en un paciente de 75 años. En todos los casos de infección, los pacientes se presentaron con comórbidos asociados, predominantemente diabetes mellitus, de éstos, cuatro

Tabla 1: Datos clínicos y epidemiológicos del grupo control con y sin vancomicina intervenidos por cirugía de columna.

Con vancomicina Sin vancomicina

\begin{tabular}{lcc}
\hline Edad (años) (DE) & 55.1 & 60.1 \\
Género & & \\
$\quad$ Masculino & 33 & 28 \\
$\quad$ Femenino & 27 & 32 \\
Nivel quirúrgico & & \\
$\quad$ Cervical & 5 & 6 \\
$\quad$ Torácico & 7 & 5 \\
$\quad$ Lumbar & 48 & 49 \\
Tipo de patología & & \\
$\quad$ Traumática & 35 & 28 \\
$\quad$ Degenerativo & 25 & 32 \\
Comórbidos & & \\
$\quad$ Hipertensión arterial & 19 & 24 \\
$\quad$ Diabetes mellitus 2 & 27 & 23 \\
$\quad$ Nefropatía, tabaquismo & 20 & 19 \\
\hline
\end{tabular}


Tabla 2: Datos clínicos y descriptivos de los pacientes que presentaron infección del sitio quirúrgico posterior a la cirugía, encontrados en el grupo control sin uso de vancomicina.

\begin{tabular}{ccccccc}
\hline \multirow{2}{*}{ Edad (años) } & Género & Tipo de infección & Comórbidos & Tipo de patología & Nivel instrumentado & Tiempo de infección \\
\hline 64 & Masculino & Superficial & HAS/DM & Degenerativa & Lumbar & Temprana \\
72 & Femenino & Superficial & HAS/DM & Degenerativa & Lumbar & Temprana \\
75 & Masculino & Profunda & DM & Degenerativa & Lumbar & Tardía \\
56 & Femenino & Superficial & HAS/DM & Degenerativa & Lumbar & Temprana \\
44 & Masculino & Superficial & Tabaquismo & Traumática & Torácico & Temprana \\
\hline
\end{tabular}

fueron mayores de 50 años y uno con una edad de 44 años. De todos los casos infectados, cuatro de ellos fueron sometidos a procedimiento quirúrgico por patología degenerativa.

Dentro del análisis estadístico, con base en las variables infección-edad encontramos un total de cuatro pacientes infectados con una edad mayor a 50 años, y sólo un paciente menor a 50 años, reportando una RR de 0.5833, un IC de 0.0675-5.0394 y una $p=0.5264$, siendo esta relación no estadísticamente significativa; sin embargo, esto indica que la edad menor de 50 es un factor protector ante la presencia de infección de sitio quirúrgico en nuestra población.

En cuanto a la correlación patología-infección, se reportaron cuatro pacientes infectados con patología degenerativa y sólo uno traumática, reportándose un RR 0.2262, IC de 0.026-1.965, $p=0.1525$, indicando que la patología traumática es un factor protector ante la infección, pero no significativo en nuestros pacientes. En relación con el segmento torácico y lumbar, encontramos que cuatro casos presentaron infección en el grupo de segmento lumbar; sin embargo, sólo un paciente presentó infección en el grupo torácico, reportándose un RR 2.0208, un IC de 0.2456-16.6295 y una $p=0.4483$, para la región lumbar en nuestra población.

\section{Discusión}

Nuestros resultados no encontraron diferencia estadísticamente significativa $(p \leq 0.05)$ en la relación entre el uso de vancomicina y la infección postquirúrgica en estos cinco únicos casos descritos (Tabla 2), en el grupo de pacientes sin vancomicina, intervenidos por cirugía de columna en el Centro Médico Nacional de Occidente Guadalajara, Jalisco.

Sweet $F$ y colaboradores en 2011 realizaron una comparación retrospectiva para evaluar la seguridad y eficacia de la aplicación local de vancomicina como complemento para la profilaxis de infecciones de heridas de la columna torácica y lumbar instrumentadas por vía posterior en comparación con terapias intravenosas solas. No hubo resultados clínicos adversos o complicaciones en la herida relacionadas con la aplicación local de vancomicina comparable con nuestros pacientes, ellos concluyeron que la aplicación local complementaria de vancomicina en polvo disminuye la tasa de infección de la herida postquirúrgica en la instrumentación posterior de la columna vertebral a diferencia del nuestro con significancia estadística $(p \leq 0.0001)$. Este estudio proporciona evidencia terapéutica de nivel III, la aplicación local complementaria de vancomicina en polvo disminuye la tasa de infección de la herida postquirúrgica en comparación con la profilaxis sola con cefalexina intravenosa en la fusión toracolumbar con instrumentación posterior. ${ }^{11}$

Joshua B y su equipo, en su análisis en 2014, refieren que las infecciones del sitio quirúrgico pueden conducir a una mayor morbilidad, mortalidad y costes sanitarios. En su estudio comparan el uso de vancomicina contra no emplearla en el desarrollo de una infección profunda de la herida (OR 0.23, 95\%, $\mathrm{IC}=0.11-0.50, p=0.0002$ ). Para infecciones superficiales y profundas combinadas, la OR $=0.43(95 \%$, $\mathrm{IC}=0.22-0.82, \mathrm{p}=0.01$ ). Entre sus conclusiones reportan la seguridad de usar polvo de vancomicina en el sitio quirúrgico. ${ }^{12}$ Entre nuestras infecciones reportamos un caso aislado de infección profunda, por lo que no se pueden realizar mediciones específicas para conocer la razón exacta.

George $\mathrm{M}$ y colaboradores, en su revisión del 2015, encontraron un total de 6,701 (68.9\%) pacientes con vancomicina en cirugía lumbar. La tasa media de infecciones entre el control y casos tratados con vancomicina fue de 7.47 y $1.36 \%$, respectivamente. Hubo un total de 23 eventos adversos: nefropatía (un paciente), ototoxicidad que resulta en una pérdida 
auditiva transitoria (dos casos), absorción sistémica que resulta en exposición terapéutica a vancomicina (una persona) y formación de seroma con cultivo negativo (19 pacientes). La tasa general de eventos adversos para el número total de sujetos tratados fue de $0.3 \%$. Ellos concluyen que el uso de vancomicina a nivel de la herida parece ser seguro y efectivo para reducir las infecciones postoperatorias con una baja tasa de morbilidad, ${ }^{13}$ entre nuestros casos no existieron complicaciones de ningún tipo asociadas al uso de vancomicina en polvo.

Abhiram G y su grupo reportaron en 2019412 casos de infección (3.8\%) en el grupo control de 10,846 pacientes en comparación con 197 de infección (2.3\%) en el grupo de vancomicina en polvo en un total de 8,456 casos. $\mathrm{OR}=0.60(95 \%, \mathrm{IC}=0.51-0.71$, $p<0.05)$. La tasa de infecciones grampositivos fue significativamente mayor en el grupo control en comparación con el grupo de vancomicina (70 vs $45.1 \%$, $p<0.05)$. La tasa de infección con gramnegativos y polimicrobianos fue significativamente mayor en el grupo de vancomicina (35.8 frente a 18.5\%, $p<0.05$ ). El riesgo de desarrollar una infección del sitio quirúrgico con gramnegativos o polimicrobianos fue casi el doble (93.5\% más alto) en el grupo de la vancomicina. Esta distinción entre el desarrollo del tipo de agente causante fue otra limitante en nuestro estudio, por los pocos casos documentados de infección en la herida y sólo un caso con infección profunda y tardía sin aislar un agente en específico. ${ }^{15}$

Podemos encontrar hallazgos comparables con Sipeng $L$ y colaboradores en el 2019, quienes describen un análisis en 15,548 pacientes; reportan que el uso tópico de polvo de vancomicina fue eficaz para reducir la incidencia de infecciones bacterianas grampositivas en sitios quirúrgicos de columna (OR $0.41, p<0.00001)$ sin afectar su eficacia en la prevención de infecciones polimicrobianas (OR 0.30, $p=0.03$ ). Además, podría reducir significativamente la tasa de infección por estafilococos resistentes a la meticilina (OR 0.34, $p<0.0001$ ). Sin embargo, el polvo de vancomicina tópica no mostró ventajas para prevenir infecciones bacterianas gramnegativas (OR $0.94, p=0.75$ ). Entre sus conclusiones refieren que la administración tópica de vancomicina en polvo puede no aumentar las tasas de bacterias gramnegativas o polimicrobianas y por el contrario pueden reducir significativamente las tasas de infección de bacterias grampositivas, estafilococos resistentes a la meticilina (MRS). Ellos asocian que el polvo de vancomicina tópica no puede cambiar las tasas de infecciones bacterianas gramnegativas por el espectro antimicrobiano de la vancomicina. ${ }^{16}$

Otras investigaciones comparan dosis de vancomicina en polvo, Sombat $\mathrm{K}$ y su grupo en Bangkok en el 2019 presentan resultados con 131 casos que fueron tratados con instrumentación posterior más fusión de la columna vertebral, utilizando cefazolina intravenosa y adyuvante $2 \mathrm{~g}$ de vancomicina polvo en la herida. Cinco pacientes de este grupo desarrollaron infecciones profundas (3.8\%). Ciento treinta y cuatro del estudio fueron tratados con cefazolina intravenosa y adyuvante $1 \mathrm{~g}$ de vancomicina en polvo. De éstos, cuatro pacientes desarrollaron infecciones profundas $(2.98 \%)$. Ciento treinta y cinco pacientes en el grupo de control fueron tratados sólo con cefazolina intravenosa como profilaxis. De estos, cuatro pacientes desarrollaron infecciones profundas $(2.96 \%)$. El estafilococo coagulasa negativo fue el agente aislado más común. No hubo resultados clínicos adversos ni complicaciones de la herida debido a la aplicación local de polvo de vancomicina. La cantidad de casos de infección reportados es similar a la nuestra de igual manera sin reportar valores significativos $(p \leq 0.05) .{ }^{17}$

En Latinoamérica, Alcalá C y colaboradores en 2013 incluyeron seis estudios controlados con un total de 3,379 pacientes. Los riesgos relativos combinados fueron: infección del sitio quirúrgico $R R=0.11$, (95\% $\mathrm{IC}=0.05-0.25, \mathrm{p}<0.00001$ ), y pseudoartrosis $\mathrm{RR} 0.87$ $(95 \%$ IC $=0.34-2.21, p=0.77)$. En 1,437 pacientes tratados no se reportaron complicaciones asociadas al uso de la vancomicina. Concluyen que la aplicación de vancomicina en polvo dentro de la herida se asoció con una reducción significativa del riesgo de infecciones del sitio quirúrgico, sin incrementar el de pseudoartrosis o de efectos adversos. ${ }^{19}$

En México, una serie de casos en 2018 de Fabian L y su grupo reportan para un total de 125 pacientes un RR de infección de herida quirúrgica de $1.5 \%$ en el grupo que recibió vancomicina y de $5.3 \%$ en el grupo control con una $p=0.24$ no estadísticamente significativa. Los factores asociados a la presentación de infecciones fueron la edad, las instrumentaciones de más de seis niveles y la prolongación del tiempo quirúrgico, aunque no se pudieron determinar valores para establecer una significancia estadística; concluyen que las infecciones tempranas en instrumentaciones de columna de su serie muestran una disminución asociada a la aplicación de vancomicina tópica en el cierre del lecho quirúrgico. Hallazgos comparables a una muestra similar a nuestros resultados con un número de casos infectados similares, cuatro de ellos 
distribuidos en sus dos grupos contra los cinco casos de infección, pero sólo en el grupo sin vancomicina y sin reportar complicaciones por el uso de ésta. ${ }^{20}$

Los casos de infección no permitieron realizar el cálculo de riesgo relativo en nuestros grupos de estudio, pero se detecta un RR de $0.5833,0.2262$ y 2.0208 en cuanto a la asociación entre una edad menor de 50 años, una patología de tipo traumática como las menos afectadas por la infección de la herida y la región lumbar, como el sitio con presencia de más casos con infección, aunque sin significancia estadística ( $p \leq 0.05)$, estos hallazgos fundamentan el realizar investigación que ofrezca alternativas a los cirujanos de columna para evitar la infección, tal es el caso del uso de vancomicina que representa un beneficio con bajo costo y un método con pocas complicaciones documentadas para los pacientes. Se requieren más estudios en poblaciones similares a la nuestra respecto al nivel socioeconómico, elevados índices de obesidad, diabetes mellitus, hipertensión arterial sistemática y tabaquismo que pueden influir en la presencia de infecciones ocasionadas por diversos microorganismos (Tabla 2); en el ámbito hospitalario, una infección que complique la evolución satisfactoria de los pacientes sometidos a cirugía de columna puede influir en los costos de atención, encareciendo un procedimiento que de entrada representa un importante gasto por cirugía y repercute en la calidad de vida. Las complicaciones y efectividad son objeto de estudio, pero existe evidencia científica que respalda el uso tópico de la vancomicina en las instrumentaciones posteriores de la columna vertebral, una alternativa adecuada en un procedimiento que se realiza cada vez con mayor frecuencia alrededor del mundo. Es necesario seguir métodos rigurosos y con muestras adecuadas para encontrar hallazgos que representen resultados significativos y concluyentes en favor del uso de la vancomicina en la herida. ${ }^{11-20}$

\section{Conclusiones}

No existe significancia estadística en nuestra investigación de que la vancomicina tópica juegue un papel fundamental en la prevención de infecciones a nivel de herida quirúrgica en la cirugía vertebral. Sin embargo, es importante notar que todas las personas infectadas en este trabajo pertenecieron al grupo B (sin uso de vancomicina). Por otra parte, evidenciamos que la patología traumática y una edad menor de 50 años pueden tener un rol protector ante la infección local en los abordajes espinales y el nivel lumbar puede ser condicionante para la presencia de infección.

\section{Agradecimientos}

Agradecimiento al equipo quirúrgico de Traumatología y Ortopedia en HE CMNO por todas las facilidades y cooperación para poder realizar este estudio.

\section{Referencias}

1. Burke A. Vancomycin. Antimicrobial therapy II. Med Clin North Am. 1995; 79: 817-831.

2. Donald P. Vancomycin: a history. Clin Infect Dis. 2006; 42: S5-S12.

3. Mark P. Vancomycin. Mayo Clin Proc. 1991; 66: 1165-1170.

4. Dennis $L$. The role of vancomycin in the treatment paradigm. Clin Infect Dis. 2006; 42: S51-S57.

5. Kanj W, Flynn J, Spiegel D, Dormans J. Vancomycin prophylaxis of surgical site infection in clean orthopedic surgery. Orthopedics. 2013; 36 (2): 138-146.

6. Melichercik P, Klapkova E, Landor I. The effect of vancomycin degradation products in the topical treatment of osteomyelitis. Bratisl Lek Listy. 2014; 115 (12): 796-799.

7. Baron J, Shamrock A, Cates W. Graft preparation with intraoperative vancomycin decreases infection after $\mathrm{ACL}$ reconstruction. J Bone Joint Surg Am. 2019; 101: 2187-2193.

8. Heckman N, Mayfield C, Culvern C. Systematic review \& meta-analysis of intrawound vancomycin in total hip and total knee arthroplasty: a call for a prospective randomized trial. J Arthroplasty. 2019; 34: 1815-1822.

9. Chen A, Fleischman A, Austin M. Use of intrawound antibiotics in orthopaedic surgery. J Am Acad Orthop Surg. 2018; 26: e371-e378.

10. O'Toole R, Joshi M, Carlini A. Local antibiotic therapy to reduce infection after operative treatment of fractures at high risk of infection: a multicenter, randomized, controlled trial (VANCO Study). J Orthop Trauma. 2017; 31 (4): S18-S24.

11. Sweet F, Roh M, Sliva C. Intra-wound application of vancomycin for prophylaxis in instrumented thoracolumbar fusions: efficacy, drug levels, and patient outcomes. Spine (Phila Pa 1976). 2011; 36 (24): 2084-2088.

12. Joshua B, Nader S, Sandi K. The use of vancomycin powder in modern spine surgery: systematic review and meta-analysis of the clinical evidence. World Neurosurg. 2015; 83 (5): 816-823.

13. George M, David W, Kim W. Complications from the use of intrawound vancomycin in lumbar spinal surgery: a systematic review. Neurosurg Focus. 2015; 39 (4): E11.

14. Hiroshi T, Yasuaki I, Yuichirou Y. Use of intrawound vancomycin powder against postoperative infection after spine surgery. Spine Surg Relat Res. 2018; 2 (1): 18-22.

15. Abhiram G, Alex R, Torin C. Selection pressures of vancomycin powder use in spine surgery: a metaanalysis. Spine J. 2019; 19 (6): 1076-1084.

16. Sipeng L, Hongtao R, Xueqin Z. Metaanalysis of topical vancomycin powder for microbial profile in spinal surgical site infections. Eur Spine J. 2019; 28 (12): 2972-2980.

17. Sombat K, Sirichai S, Chaiwat P. Comparison between $1 \mathrm{~g}$ and $2 \mathrm{~g}$ of intrawound vancomycin powder application for prophylaxis in posterior instrumented thoracic or lumbosacral spine surgery: a preliminary report. Asian J Neurosurg. 2019; 14: 710-714. 
18. Justin V, Cumhur O, Sebastiaan P. The efficacy of intrawound vancomycin powder and povidone-iodine irrigation to prevent surgical site infections in complex instrumented spine surgery. Spine J. 2019; 19 (10): 1648-1656.

19. Alcalá G, Paternina A, Moscote L. Aplicación de vancomicina en polvo dentro de la herida quirúrgica durante cirugías de columna: revisión sistemática y metaanálisis. Rev Esp Cir Ortop Traumatol. 2014; 58 (3): 182-191.

20. Fabian L, Félix S, Martín G. Vancomicina tópica en la prevención de infecciones tempranas en cirugías instrumentadas espinales: serie de casos y revisión de la literatura. Rev Argent Neuroc. 2018; 32 (2): 158-164.

\section{Conflicto de intereses}

Sin conflicto de intereses por parte de los autores de este documento. 